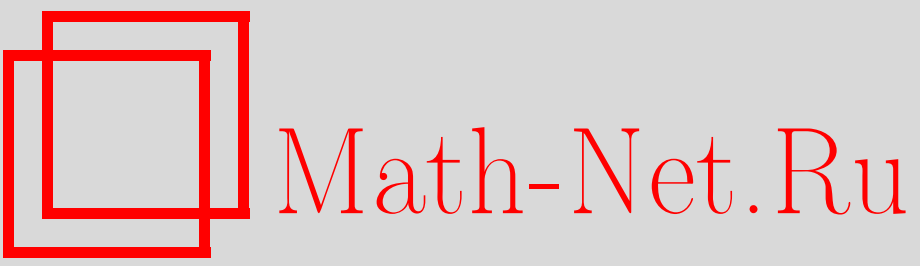

М. В. Половинкина, И. П. Половинкин, С. А. Рабееах, Об эффекте Гюйгенса и точках безнадежности в макроэкономической модели Т. Пу, Итоги науки и техн. Сер. Соврем. мат. и ее прил. Темат. обз., 2021, том 191, 129-134

DOI: https://doi.org/10.36535/0233-6723-2021-191-129-134

Использование Общероссийского математического портала Math-Net.Ru подразумевает, что вы прочитали и согласны с пользовательским соглашением

http: //www. mathnet.ru/rus/agreement

Параметры загрузки:

IP: 3.95 .254 .165

26 апреля 2023 г., 13:24:00 
ИТОГИ НАУКИ И ТЕХНИКИ.

Современная математика и ее приложения.

Тематические обзоры.

Том 191 (2021). C. 129-134

DOI: 10.36535/0233-6723-2021-191-129-134

УДК 517.956.326, 519.862.5

\title{
ОБ ЭФФЕКТЕ ГЮЙГЕНСА И ТОЧКАХ БЕЗНАДЕЖНОСТИ В МАКРОЭКОНОМИЧЕСКОЙ МОДЕЛИ Т. ПУ
}

\author{
(c) 2021 г. \\ М. В. ПОЛОВИНКИНА, И. \\ и. П. половинкин, с. \\ C. A. PAБEEAX
}

\begin{abstract}
АннотАция. Рассматривается макроэкономическая модель Т. Пу, описывающая колебания валового дохода в заданном регионе. При особом сочетании норм сбережений и инвестирования, отклонения дохода будут иметь место лишь в течение конечного промежутка времени, после которого доход вернется к стационарному состоянию. Этот эффект известен в математической физике как принцип Гюйгенса. Результаты исследования модели с помощью статистических методов анализа данных позволяют говорить о правдоподобности гипотезы о наличии эффекта Гюйгенса в определенные периоды истории российской экономики. Рассмотрен вопрос о строении множества стационарных нулей нетривиальных решений стационарного уравнения.
\end{abstract}

Ключевые слова: модель распределения дохода, принцип Гюйгенса, задача Коши, область зависимости, стационарные нули решения.

\section{ON THE HUYGENS EFFECT AND POINTS OF HOPELESSNESS IN THE T. PUU MACROECONOMIC MODEL}

\author{
(c) 2021 M. V. POLOVINKINA, I. P. POLOVINKIN, S. A. RABEEAKH
}

\begin{abstract}
We consider the macroeconomic model proposed by T. Puu, which describes fluctuations in gross income in a given region. Under a special combination of savings and investment rates, income deviations occur only for a finite period of time, after which the income returns to a stationary state. In mathematical physics, this effect is called the Huygens effect (principle). Results of the study of the model by statistical methods allow one to speak about the plausibility of the hypothesis about the presence of the Huygens effect in certain periods of the history of the Russian economy. The question on the structure of the set of stationary zeros of nontrivial solutions of a stationary equation is considered.
\end{abstract}

Keywords and phrases: income distribution model, Huygens principle, Cauchy problem, dependence domain, stationary zeros of the solution.

AMS Subject Classification: 35L81, 91B64

1. Введение. В теории гиперболических уравнений в частных производных, описывающих волновые процессы, известно явление под названием «принцип Гюйгенса». Мы придерживаемся его геометрического определения по Ж. Адамару в терминологии И. Г. Петровского (см. [18, c. 353-354]). Задача Коши для гиперболического уравнения удовлетворяет принципу Гюйгенса, если для каждой точки пространства размерность области зависимости решения от начальных данных (которая определяется как дополнение к объединению всех лакун) меньше размерности пространства. Это явление имеет место для трехмерного волнового уравнения, описывающего распространение звука и света. Оно проявляется в том, что колебания, вызванные локализованным источником, происходят в течение конечного промежутка времени без последействия, так 
что волна имеет резкий задний и резкий передний фронты. Экономическая теория признает волнообразный характер развития экономических систем. В связи с этим представляется уместным задаться вопросом о возможности проявления принципа Гюйгенса в экономике.

2. Исходные предположения и основное уравнение модели. Следуя [21], обозначим через $Y=Y(x, y, t)$ отклонение уровня дохода от стационарного состояния в точке $(x, y)$ в момент времени $t$. Если быть более точным, то $Y=Y(x, y, t)$ - это вклад в валовой внутренний продукт (ВВП), однако мы используем терминологию [21]. Будем считать, что сбережения $S$ находятся в заданном отношении $s=s(t)$ к доходу. Пусть $v=v(t)$ означает отношение между основным капиталом и доходом. Инвестиции, обозначаемые $I$, по определению являются темпами изменения основного капитала. Будем также предполагать, что существует некоторый адаптивный процесс, при котором доходы возрастают пропорционально разности составляющей, пропорциональной инвестициям и сбережениям: $\dot{Y} \sim(\alpha I-S)$. Подобную задержку предположим и при регулировании инвестиций, так что $\dot{I} \sim(v \dot{Y}-\beta I)$. Итак, модель строится из следующих предположений:

$$
\begin{gathered}
I=v \frac{\partial Y}{\partial t}, \\
S=s \frac{\partial Y}{\partial t}, \\
\frac{\partial Y}{\partial t}=\alpha I-s Y, \\
\frac{\partial I}{\partial t}=v \frac{\partial Y}{\partial t}-\beta I .
\end{gathered}
$$

Взяв производную по времени от обеих частей равенства (3), получим:

$$
\ddot{Y}=\dot{\alpha} I+\alpha \dot{I}-\dot{s} Y-s \dot{Y}
$$

Выражая $\dot{I}$ из (4) и подставляя в (5), получим:

$$
\ddot{Y}=(\alpha v-s) \dot{Y}-\dot{s} Y+(\dot{\alpha}-\alpha \beta) I .
$$

Теперь, выражая $I$ из (3), мы окончательно получим уравнение дохода:

$$
\alpha \ddot{Y}-\left(\alpha^{2} v-\alpha s+\dot{\alpha}-\alpha \beta\right) \dot{Y}+(\alpha \beta s+\alpha \dot{s}-\dot{\alpha} s) Y=0 .
$$

В уравнение (7) географические координаты входят как простые параметры, так что (7) является обыкновенным дифференциальным уравнением. Если же к предположениям (3)-(4) добавить предположение о межрегиональной торговле, то в первом приближении активное торговое сальдо определяется выражением

$$
m \Delta Y=m\left(\frac{\partial^{2} Y}{\partial x^{2}}+\frac{\partial^{2} Y}{\partial y^{2}}\right)
$$

где $m$ - коэффициент пропорциональности, характеризующий склонность к импортированию. При этих предположениях уравнение (7) заменится уравнением дохода в частных производных:

$$
\alpha \ddot{Y}-\left(\alpha^{2} v-\alpha s+\dot{\alpha}-\alpha \beta\right) \dot{Y}+(\alpha \beta s+\alpha \dot{s}-\dot{\alpha} s) Y=m \Delta Y .
$$

В [21] предполагалось, что коэффициенты $s, v$ постоянны, $\alpha=\beta=1$, так что уравнение (9) записывалось в виде

$$
\frac{\partial^{2} Y}{\partial t^{2}}-(v-1-s) \frac{\partial Y}{\partial t}+s Y=m \Delta Y .
$$

3. Проявление эффекта Гюйгенса. Рассмотрим особый частный случай задания коэффициентов в уравнении (9), а именно, считая $\alpha$ и $\beta$ постоянными, положим

$$
\begin{aligned}
& s=s(t)=s_{0} e^{-\beta t} \\
& v=v(t)=\frac{s_{0}}{\alpha} e^{-\beta t}+\frac{\beta}{\alpha}-\frac{1}{\alpha t} .
\end{aligned}
$$


При таком выборе темпов инвестиций и сбережений уравнение (9) примет вид

$$
\frac{\partial^{2} Y}{\partial t^{2}}+\frac{1}{t} \frac{\partial Y}{\partial t}=\frac{m}{\alpha} \Delta Y
$$

Уравнение (13) - сингулярное уравнение, известное как уравнение Эйлера-Пуассона-Дарбу (см. [22] и обзор там). Левая часть этого уравнения содержит оператор Бесселя $B_{\gamma}=\partial^{2} / \partial t^{2}+$ $\gamma / x \partial / \partial t, \gamma=1$. Сингулярные уравнения в частных производных с оператором Бесселя изучались И. А. Киприяновым и его учениками (см. [13-15, 17, 22]).

Следует отметить качественную особенность, возникшую при рассмотрении уравнений типа (13): появление принципа Гюйгенса для уравнения с четным числом пространственных переменных (см. по этому поводу $[3-12,16])$. В нашем случае это легко видеть. Непосредственной проверкой нетрудно убедиться, что функция

$$
Y(x, y, t)=\frac{1}{2 \pi t \sqrt{m / \alpha}} \oint_{S_{\sqrt{m / \alpha} t}(x, y)} \varphi(\xi, \eta) d l_{\xi \eta}=\frac{1}{2 \pi} \int_{-\pi}^{\pi} \varphi\left(x+\sqrt{\frac{m}{\alpha}} t \cos \theta, y+\sqrt{\frac{m}{\alpha}} t \sin \theta\right) d \theta,
$$

где $S \sqrt{m / \alpha} t(x, y)$ - окружность на плоскости с центром в точке $(x, y)$ и радиусом $\sqrt{m / \alpha} t$, является регулярным решением уравнения (13) и удовлетворяет начальным условиям

$$
\lim _{t \rightarrow+0} Y(x, y, t)=\varphi(x, y), \quad \lim _{t \rightarrow+0} \frac{\partial Y}{\partial t}(x, y, t)=0 .
$$

Обратим теперь внимание на следующую особенность в задании функции (14): для определения значения отклонения дохода $Y$ в точке $(x, y)$ в момент времени $t$ достаточно задать значения функции $\varphi(x, y)$ лишь на окружности $S \sqrt{m / \alpha} t(x, y)$, а открытый круг с границей $S \sqrt{m / \alpha} t(x, y)$ будет являться лакуной, т.е. множеством точек, изменение начальных данных на котором не повлечет за собой изменения решения в точке $(x, y)$ в момент времени $t$. Лакуной будет и внешняя часть этого круга. Другими словами, областью зависимости решения задачи Коши (13), (15), (??) в точке $(x, y)$ в момент времени $t$ является окружность $S_{\sqrt{m / \alpha} t}(x, y)$, так что размерность области зависимости решения от начальных данных меньше размерности многообразия начальных данных, то есть имеет место принцип Гюйгенса (см. $[1,2,19]$. С точки зрения рассматриваемого здесь приложения это означает следующее. Начальное отклонение дохода, локализованное на плоскости, повлечет за собой в точках плоскости отклонение дохода, локализованное во времени, то есть отклонения дохода будут иметь место лишь в течение конечного промежутка времени, после которого доход вернется к стационарному состоянию. Но тогда подобная ситуация означала бы своего рода тщетность усилий этих субъектов. Мы оставляем в стороне вопрос о возможности создания условий, при которых входные данные могли бы определяться формулами (11) и (12). Нам представляется, что при определенном влиянии некоторого центра на поведение субъектов, участвующих в товарообмене, эти условия вполне реализуемы. Это может стать своего рода управляющим фактором. Рассматриваемая модель не позволяет дать ответ на вопрос о целях и мотивации центра при создании таких условий. Одним из примеров такой мотивации может служить ускорение какой-либо экономической активности, обусловливающей увеличение налоговой составляющей доходной части бюджета вне зависимости от повышения благосостояния. Разумеется, не исключается и случай, когда подобная динамика является случайным продуктом развития рыночной экономики. То, что норма сбережений задается формулой (11), в дискретном измерении с промежутком в один год должно проявиться таким образом, что годовые значения нормы сбережений составляли бы геометрическую прогрессию. Разумеется, в точности это невозможно. На основе качественного экономического анализа можно полагать, что такая динамика нормы сбережений имела место для России в 1993-1998 гг. Конечно, этот период является довольно коротким, но он характеризует определенный этап в развитии экономики России. Используя данные Росстата о ВВП (см. сайт Федеральной службы государственной статистики), валовых сбережениях и инвестициях за указанный период, мы рассчитали темп падения нормы сбережений, и оказалось, что она падала примерно в геометрической прогрессии (мы получили 
адекватную модель с коэффициентом детерминации $\left.R^{2}=0,89\right)$. Это позволяет говорить о возможном наличии эффекта Гюйгенса в отечественной экономике в 1993-1998 гг. Аналогичный анализ данных для некоторых других стран бывшего СССР (например, для Казахстана) за те же годы не позволил предположить наличие эффекта Гюйгенса в их экономике.

Укажем еще на один случай, когда имеет место принцип Гюйгенса. Здесь мы предположим, что и склонность к импортированию $m$ тоже зависит от времени, причем $m=m(t) \neq 0, t>0$. Запишем уравнение распределения дохода в виде

полагая

$$
a(t) \frac{\partial^{2} Y}{\partial t^{2}}+b(t) \frac{\partial Y}{\partial t}+\frac{\left(s(t)+s^{\prime}(t)\right)}{m(t)} Y=\Delta Y, \quad t>0,
$$

$$
\mu(t)=s(t)+1-v(t), \quad a(t)=\frac{1}{m(t)}, \quad b(t)=\frac{\mu(t)}{m(t)} .
$$

Пусть теперь

Тогда в уравнении (16)

$$
s(t)=v(t)=s_{0} e^{-t}, \quad m(t)=1+\frac{1}{t} .
$$

$$
a(t)=b(t)=\frac{t}{t+1}, \quad s(t)+s^{\prime}(t)=0 .
$$

Введем в рассмотрение функцию

$$
Y(x, y, t)=\frac{1}{2 \pi t e^{t}} \oint_{S_{t}(x, y)} \varphi(\xi, \eta) d l=\frac{1}{2 \pi e^{t}} \int_{-\pi}^{\pi} \varphi(x+t \cos \theta, y+t \sin \theta) d \theta .
$$

Непосредственной подстановкой можно убедиться в том, что функция, определенная формулой (20), удовлетворяет уравнению (16) с коэффициентами, определенными формулами (19), а также начальным условиям

$$
\lim _{t \rightarrow 0+} Y(x, y, t)=\varphi(x, y), \quad \lim _{t \rightarrow 0+}\left(t \frac{\partial Y(x, y, t)}{\partial t}\right)=0 .
$$

Рассматриваемая нами линейная модель, безусловно, упускает многие нюансы динамики в предметной области. Кроме того, уравнение в частных производных, удовлетворяющее принципу Гюйгенса, - явление достаточно редкое. В нашем случае эта «редкость» проявляется в том, что даже небольшое отклонение величин $s$ и $v$ от вида (11)-(12) приведет к утрате эффекта Гюйгенса. На наш взгляд, исследование эффекта Гюйгенса в экономике могло бы помочь в изучении влияния управляющих воздействий на реакцию экономической системы в краткосрочном и долгосрочном аспектах, в том числе в анализе отдельных макроэкономических рынков. В частности, принимая во внимание закон Оукена, связывающий колебания уровня безработицы с уровнем ВВП, мы имеем все основания полагать, что принцип Гюйгенса в случае его проявления в колебаниях ВВП проявится и в колебаниях на рынке труда.

4. Стационарное уравнение Т. Пу. Полагая, что в некоторой области $\Omega \subset \mathbb{R}^{2}$ выполняются условия

$$
\frac{\partial Y}{\partial t}=0, \quad \frac{\partial^{2} Y}{\partial t^{2}}=0, \quad \alpha=\text { const }, \quad \beta=\text { const }, \quad s=\text { const }, v=\text { const },
$$

мы получим, что уравнение (9) примет вид

$$
m \Delta Y-\alpha \beta s Y=0 .
$$

Уравнение (23) описывает стационарное распределение дохода, не зависящее от времени.

Назовем точку $\left(x^{0}, y^{0}\right)$ точкой безнадежности модели Т. Пу $(23)$, если

$$
Y\left(x^{0}, y^{0}\right)=0, \quad \nabla Y\left(x^{0}, y^{0}\right)=0 .
$$

Равенства (24) следует расценивать как отсутствие отклонения от фиксированного уровня дохода и отсутствие тенденции к отклонению. Это оправдывает принятое нами название.

Множество всех точек безнадежности назовем множеством безнадежности модели Т. Пу. 
В [20] было доказано следующее утверждение.

Теорема 1. Множество стационарных нулей нетривиального решения эллиптического уравнения

$$
P(x, D) u \equiv \sum_{|\alpha| \leqslant 2} p_{\alpha}(x) D^{\alpha} u=0, \quad x \in \Omega \subseteq \mathbb{R}^{n}, \quad n \geqslant 3,
$$

где $p_{\alpha} \in C^{\infty}(\Omega), \alpha \in(\mathbb{N} \cup\{0\})^{n},|\alpha|=\alpha_{1}+\ldots+\alpha_{n}, D^{\alpha}=\partial^{|\alpha|} /\left(\partial x_{1}^{\alpha_{1}} \ldots \partial x_{n}^{\alpha_{n}}\right)$, в окрестности любого стационарного нуля содержится в стратифицированном многообразии

$$
G=\bigcup_{k=0}^{r} M^{k}
$$

где $M^{k}(k=0,1, \ldots, r \leqslant n-2)$ - вещественно аналитическое многообразие размерности $k$. В частности, при $n=3$ пересечение достаточно малой окрестности стационарного нуля $x^{0}$ с множеством всех прочих стационарных нулей представляет собой конечное множество промежутков с общим концом в точке $x^{0}$. При $n=2$ множество стационарных нулей нетривиального решения эллиптического уравнения (25) может содержать только изолированные точки.

В частности, отсюда следует, что множество безнадежности модели Т. Пу может состоять лишь из изолированных точек безнадежности.

\section{СПИСОК ЛИТЕРАТУРЫ}

1. Азарнова Т. В., Гоголева Т. Н., Половинкин И. П., Рабееах С. А., Щепина И. Н. Об эффекте Гюйгенса в непрерывной модели распределения дохода// Вестн. Тамбов. ун-та. Сер. Естеств. техн. науки. 2016. - 21, № 6. - С. 2143-2144.

2. Азарнова Т. В., Половинкин И. П., Рабееах С. А., Щепина И. Н. Обсуждение принципа Гюйгенса в рамках макроэкономической модели Т. Пу// Мат. 39 Междунар. науч. шк.-семин. «Системное моделирование социально-экономических процессов» (ред. В. Г. Гребенников, И. Н. Щепина), 2016. C. $468-472$.

3. Зайцев В. А. О принципе Гюйгенса для некоторых уравнений с особенностями// Докл. АН СССР. 1978. - 242, № 1. - C. 28-31.

4. Зайцев В. А. О принципе Гюйгенса порядка $(p, q) / /$ в кн.: Динамика жидкости со свободными границами. - Новосибирск: Ин-т мат. Сиб. отд. АН СССР, 1980. - С. 160-165.

5. Зайщев B. А. О существовании редких волн для одной системы первого порядка// в кн.: Некоторые вопросы теории управления движением. - Саранск, 1980. - С. 69-71.

6. Зайчев B. А. Слабые лакуны для одномерных строго гиперболических уравнений// в кн.: Корректные краевые задачи для неклассических уравнений математической физики. - Новосибирск: Ин-т мат. Сиб. отд. АН СССР, 1981. - С. 66-68.

7. Зайчев B. А. Слабые лакуны для одномерных уравнений с постоянными коэффициентами// Сиб. мат. ж. -1984 . -25 , № 4 . - C. $54-62$.

8. Иванов Л. А. О принципе Гюйгенса в четномерном пространстве для некоторых уравнений с особенностями // Укр. мат. ж. - 1979. - 31, № 5. - С. 547-550.

9. Иванов Л. А. Уравнения Эйлера-Пуассона-Дарбу на сфере и их гюйгенсовость// в кн.: Корректные краевые задачи для неклассических уравнений. - Новосибирск: Ин-т мат. Сиб. отд. АН СССР, 1981. - C. $69-71$.

10. Иванов Л. А. О формулах Кирхгофа в симметрических пространствах ранга $1 / /$ Докл. АН СССР. 1981. -247 , № 4. - С. 783-785.

11. Иванов Л. А. Задача Коши для некоторых операторов с особенностями// Диффер. уравн. - 1982. 18, № 6. - C. 1020-1028.

12. Иванов Л. А. О фундаментальных решениях некоторых сингулярных уравнений// в кн.: Дифференциальные уравнения и их применения. - Вильнюс, 1982. - 32. - С. 31-45.

13. Катрахов В. В., Ситник С. М. Метод преобразования и краевые задачи для сингулярных эллиптических уравнений// Совр. мат. Фундам. напр. - 2018. - 64, № 2. - С. 211-426. 
14. Киприянов И. А. Преобразование Фурье-Бесселя и теоремы вложения для весовых классов// Тр. Мат. ин-та им. В. А. Стеклова. - 1967. - 89. - С. 130-213.

15. Киприянов И. А. Сингулярные эллиптические краевые задачи. - М.: Наука, 1997.

16. Киприянов И. А., Иванов Л. А. Задача Коши для уравнения Эйлера-Пуассона-Дарбу в однородном симметрическом римановом пространстве, I// Тр. Мат. ин-та им. В. А. Стеклова. - 1984. - 170. C. $139-147$.

17. Ляхов Л. Н. Весовые сферические функции и потенциалы Рисса, порожденные обобщенным сдвигом. - Воронеж: ВГТА, 1997.

18. Петровский И. Г. Избранные труды. Системы уравнений с частными производными. Алгебраическая геометрия. О диффузии волн и лакунах для гиперболических уравнений. - М.: Наука, 1986.

19. Половинкин И. П. К исследованию линейной модели Т. Пу динамики доходов с учетом межрегиональной торговли // Диффер. уравн. - 2012. - 48, № 6. - С. 902-903.

20. Половинкин И. П. К исследованию линейной модели Т. Пу динамики доходов с учетом межрегиональной торговли // Диффер. уравн. - 2013. - 49, № 1. - С. 132-136.

21. Пу Т. Нелинейная экономическая динамика. - Ижевск: Изд. дом «Удмуртский университет», 2000.

22. Ситник C. М., Шишкина Э. Л. Метод операторов преобразования для дифференциальных уравнений с операторами Бесселя. - М.: Физматлит, 2019.

Половинкина Марина Васильевна

Воронежский государственный университет инженерных технологий

E-mail: polovinkina-marina@yandex.ru

Половинкин Игорь Петрович

Воронежский государственный университет

E-mail: polovinkin@yandex.ru

Рабееах Светлана Александровна

Воронежский государственный университет

E-mail: srabeeakh@mail.ru 